\title{
Investigating the solid-state properties of drug-loaded mesoporous silica
}

\author{
Robert Ahern
}

School of Pharmacy, UCC

\section{Introduction}

"The drugs don't always work": Nearly 40\% of new drug compounds developed nowadays have poor water-solubility. Solubility refers to the amount of drug that will dissolve in a liquid. This means that these drugs will not easily dissolve in water; they are a bit like sand. Imagine taking a tablet for a headache that had poor water-solubility; it would take a long time to dissolve in the body and may not relieve the pain. This is a serious problem for the pharmaceutical industry because it is spending a lot of money developing drugs that cannot be brought to market. My work aims to address this challenge and develop a solution to this problem.

\section{Outline of my Work}

In my work, the anti-cholesterol drug fenofibrate will be used as the model poorly-water soluble drug. I will use a carrier material called mesoporous silica to increase the dissolution rate of fenofibrate by combining them together. The dissolution rate refers to how quickly something dissolves in a liquid over a given time; in this case, it refers to how quickly a drug dissolves in the body and works. The addition of mesoporous silica will make the fenofibrate dissolve much faster in water, like sugar in a cup of tea.

Mesoporous silica is a material that has many small pores in its structure, which range in size from 2 to 30 nanometre. The structure of mesoporous silica is highly ordered with long hexagonal pores as seen in Fig. 1. Its structure somewhat resembles the structure of honeycomb Fig. 2. and, as a result, it has a huge surface area. One gram of mesoporous silica has an area equivalent to one tenth of a football pitch. This is one of the reasons why mesoporous silica increases fenofibrate's dissolution rate, because materials with high surface areas have more contact points at which to dissolve into the liquid. 


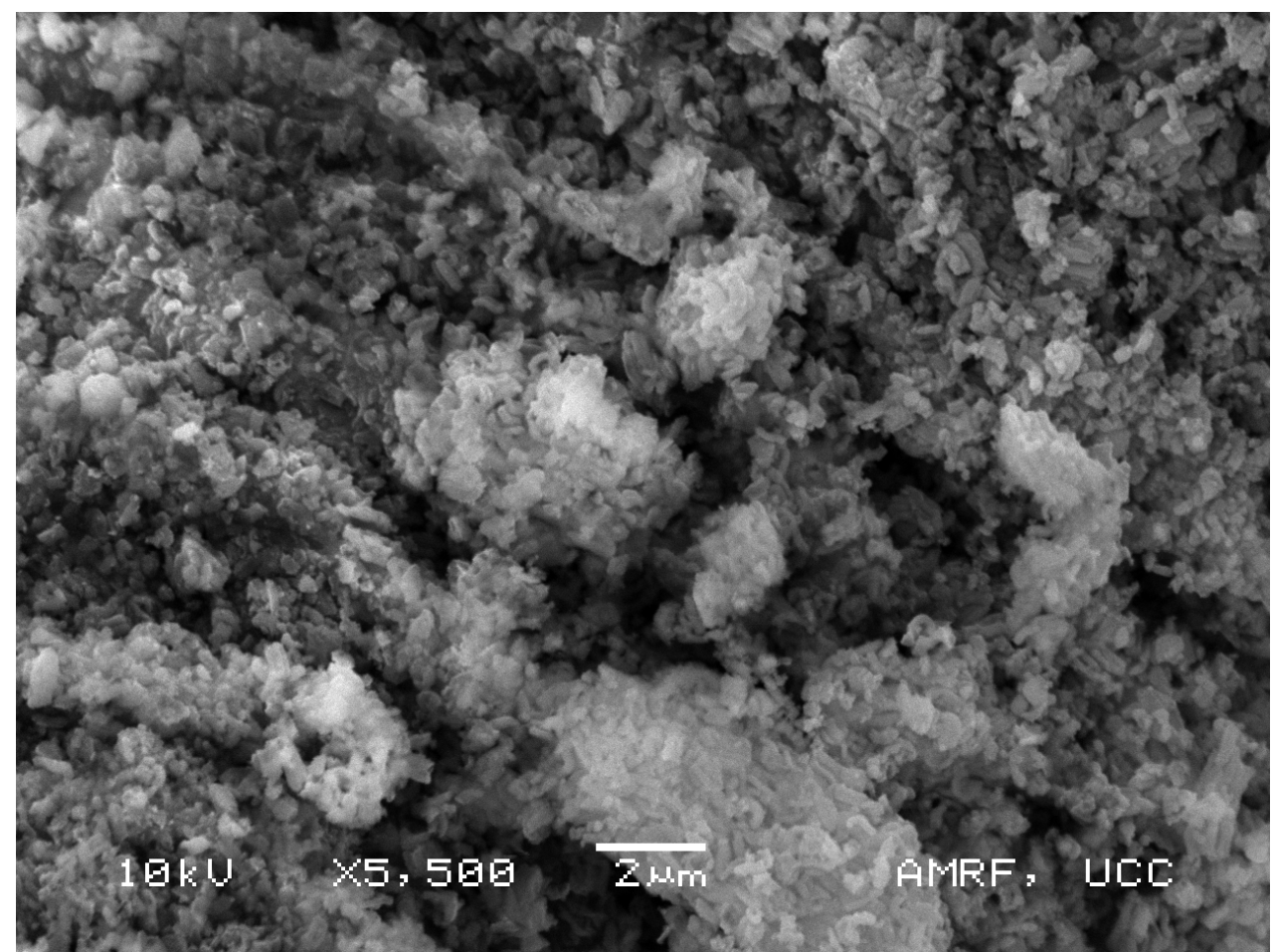

Figure 1: Electronic Microscope Image of Mesoporous Silica

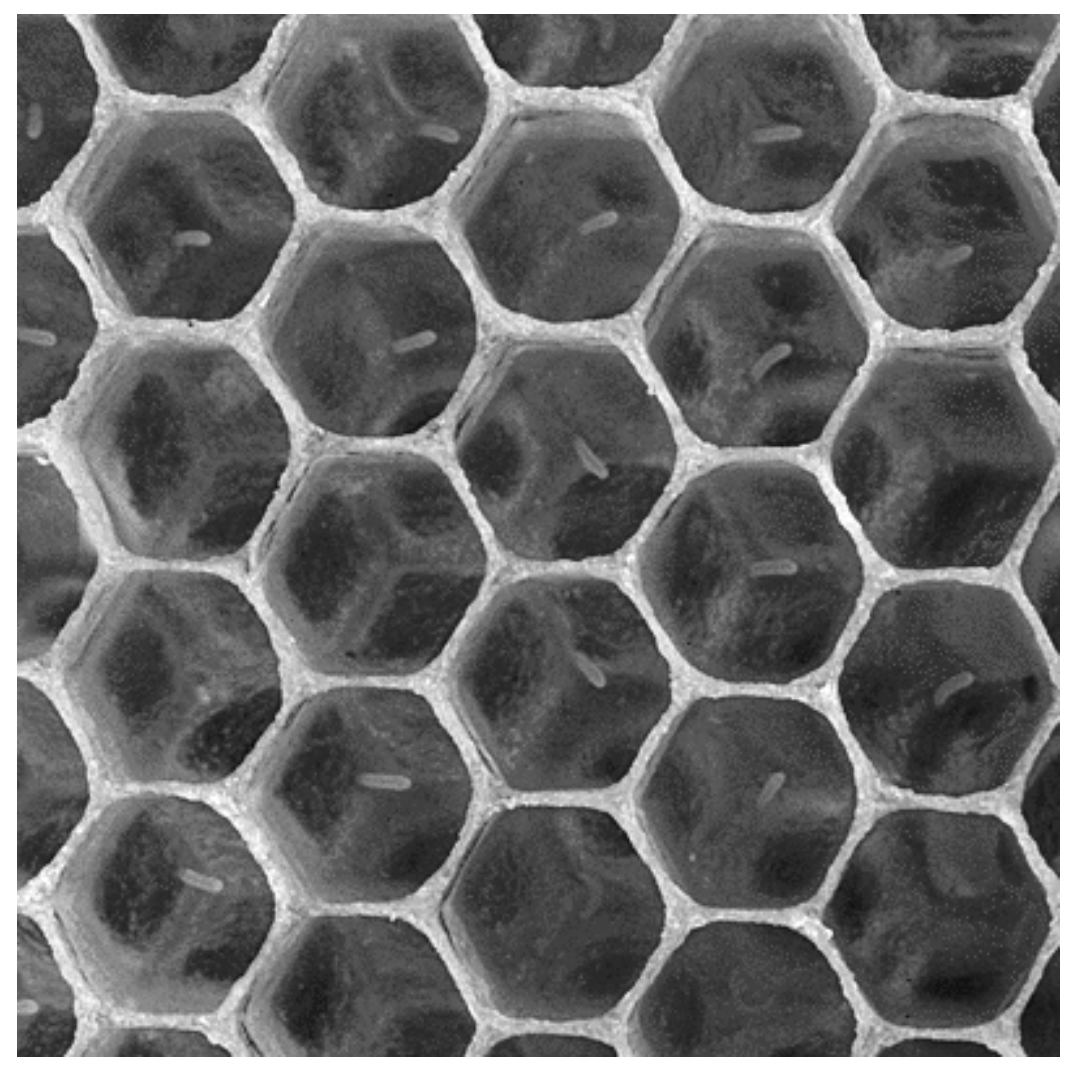

Figure 2: Structure of Honeycomb 


\section{Work Undertaken to Date}

Thus far in my work, I have combined mesoporous silica with fenofibrate in different ratios using two different methods to load the drug. The first method investigated involved physically blending them together; the two materials were put in a container and spun around just like clothes in a tumble dryer. These samples are called the physical samples. The second method I used to combine these materials was melting the fenofibrate onto and into mesopores of the silica after the initial blending step. In other words, the two materials were blended, then heated in an oven at $85^{\circ} \mathrm{C}$ for 24 hours and then cooled for 24 hours. By heating both materials to $85^{\circ} \mathrm{C}$, the fenofibrate melted and flowed into the pores and onto the surface of the mesoporous silica. These samples are called the fusion samples.

\section{Techniques Used to Characterise my Results}

Various characterisation techniques were used to determine the effect on fenofibrate of combining it with mesoporous silica. These techniques included DSC, FT-IR, P-XRD and Dissolution Studies. DSC (differential scanning calorimetry) is a thermodynamic technique that works by heating materials, usually up to and beyond their melting points. This is a very useful technique because it provides a great deal of information on a material such as its melting point and enthalpy of fusion (this is the energy required to melt one gram of it) which can be used to determine a materials structural arrangement, crystallinity. The term crystallinity refers to the solid structure of a material; essentially it refers to how structured or ordered definite shape the molecules (the building blocks of the solid material) are. P-XRD (powder x-ray diffraction) provides information on the solid state of a material. The different solid structures of a material can be identified which means that we can see if a material has a structure that is highly ordered (like building blocks of a wall) or if it is disordered. FTIR (Fourier transform infrared spectroscopy) is a technique which provides information on the chemical bonds of a material; FT-IR can be used to determine what interactions are occurring between the two materials. Dissolution studies involve dissolving a known amount of material in a suitable liquid and taking samples of the resulting mixture at different times. These samples are then used to calculate how much of the material has dissolved at each period of time. Essentially, dissolution studies can be used to try and replicate what happens in the human body when a drug is taken by a person. 


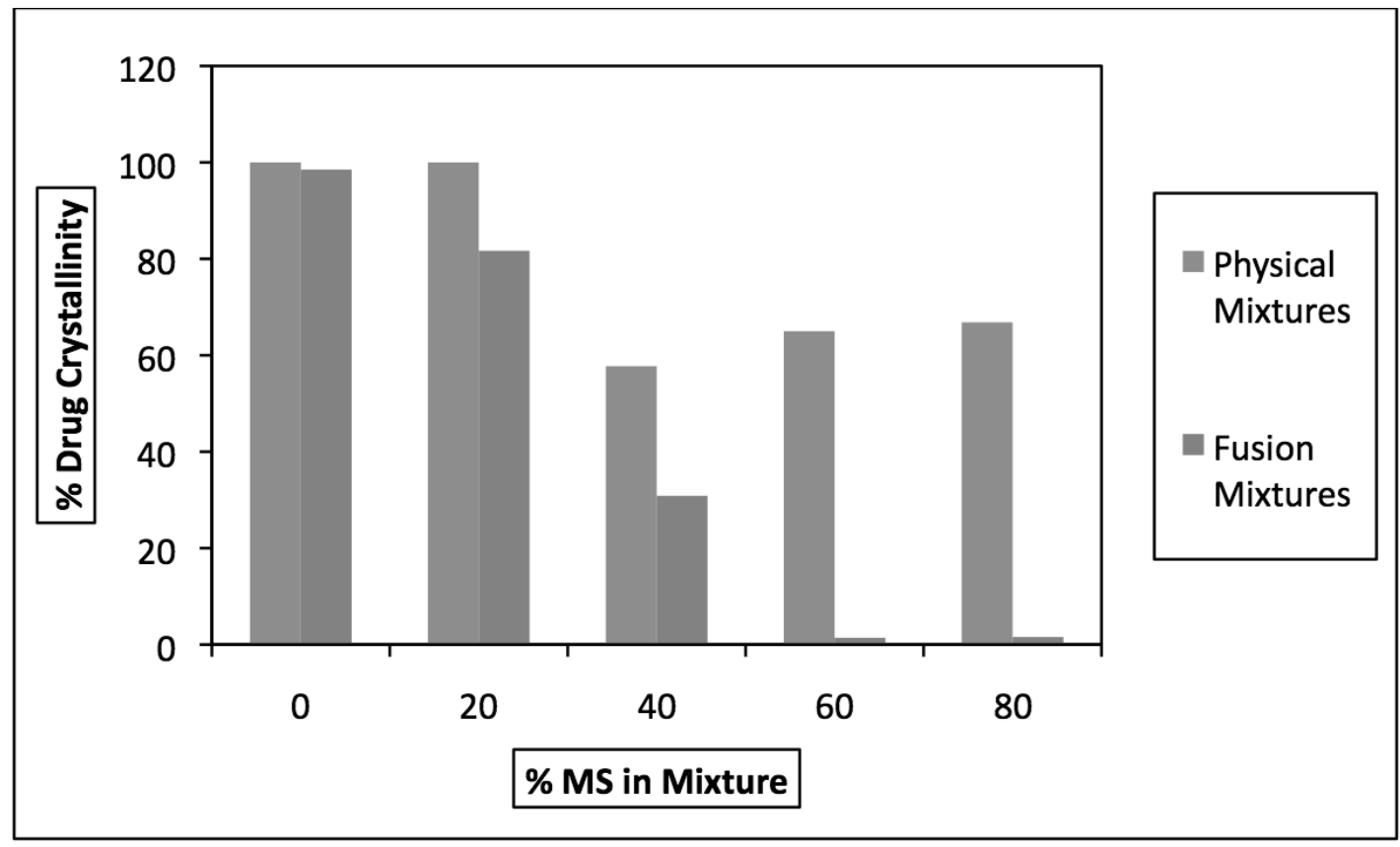

Figure 3: Effect of Addition of Mesoporous Silica on the Crystallinity of Fenofibrate.

\section{Results to Date}

\section{DSC Studies}

When mesoporous silica was added to fenofibrate, the crystallinity of fenofibrate was reduced. This reduction is most apparent in the fusion/ melting samples compared to the physical blending samples. It is clear that the mesoporous silica has altered the solid structure of the fenofibrate.

\section{P-XRD Studies}

$\mathrm{XRD}$ can provide information on the effect of the addition of mesoporous silica on the crystallinity of fenofibrate. Crystallinity refers to how ordered the structure of material is. The characteristic peaks of fenofibrate are clearly displayed.

It can be seen that the addition of mesoporous silica altered the crystallinity of fenofibrate in the physical samples. The characteristics peals of fenofibrate are reduced and changed in the physical sample. In the fusion sample, the characteristic peak of fenofibrate had disappeared. This is because fenofibrate is now in the amorphous state and therefore has a disordered structure. 


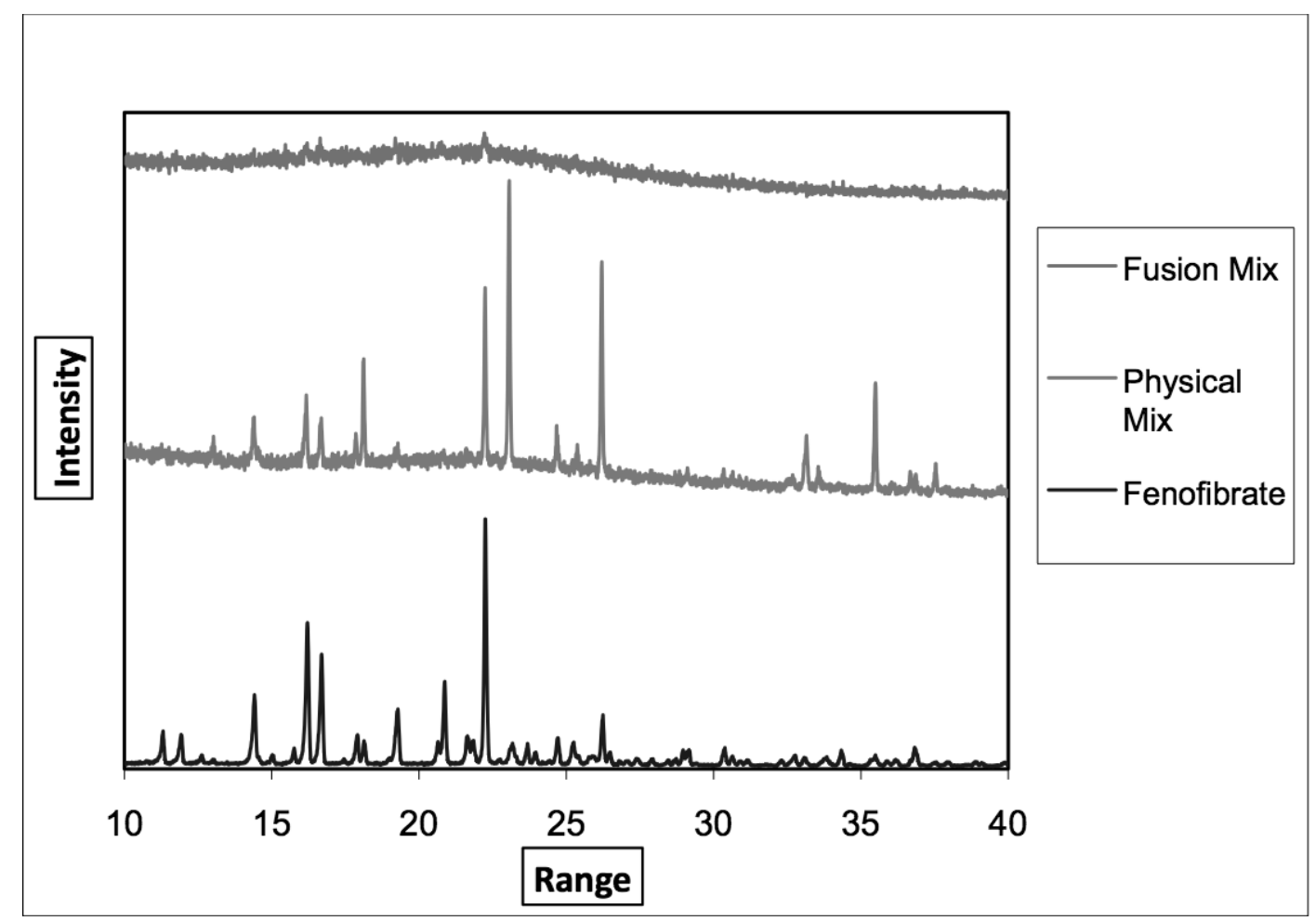

Figure 4: Effect of Addition of Mesoporous Silica on the Crystallinity of Fenofibrate.

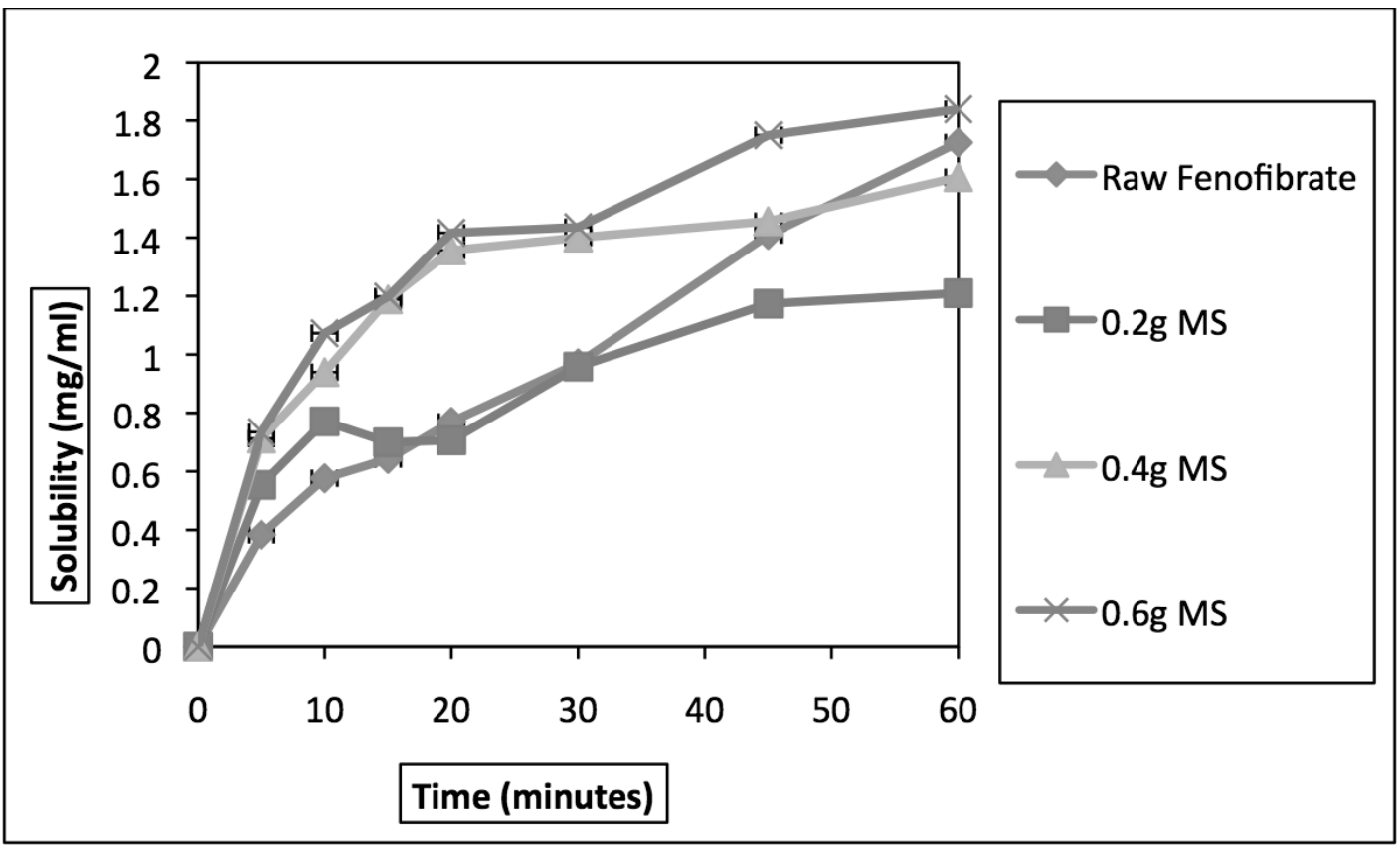

Figure 5: Enhancement of Dissolution Rate of Fenofibrate in Physical Samples. 


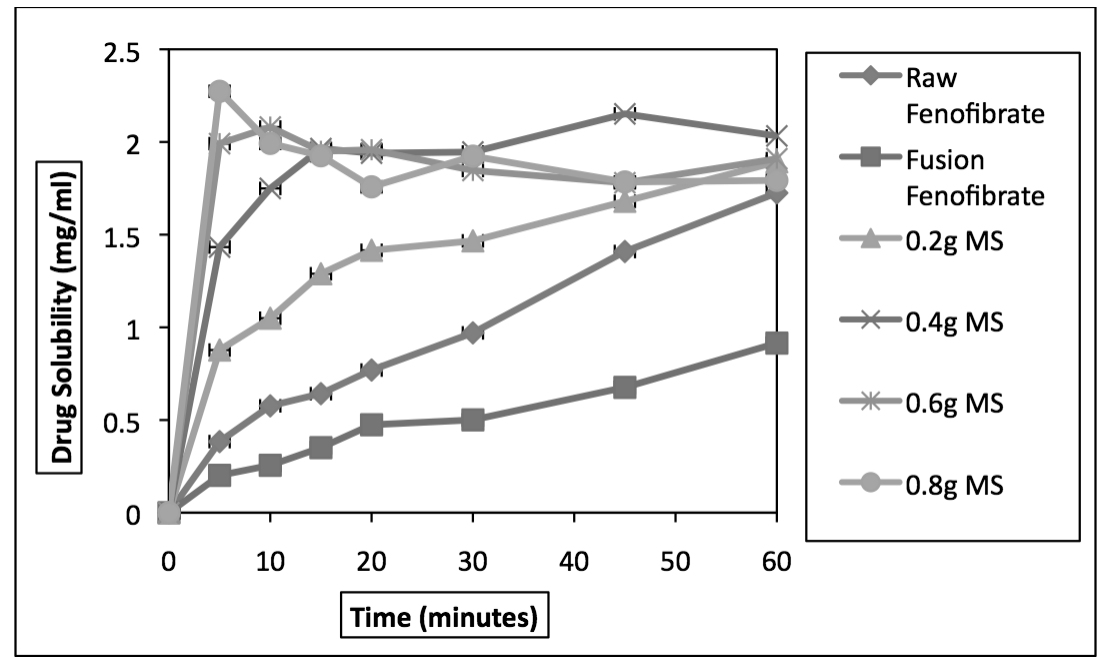

Note: MS refers to mesoporous silica in $1 \mathrm{~g}$ of sample.

Figure 6: Enhancement of Dissolution Rate of Fenofibrate in Fusion Samples.

\section{Dissolution Studies}

The addition of mesoporous silica to fenofibrate enhanced the dissolution rate of fenofibrate. This enhancement was most apparent in the fusion/melting samples and at the higher ratios of silica to drug. Thus, combining mesoporous silica with fenofibrate resulted in faster release of drug over a given time compared to the drug on its own.

\section{Discussion and Conclusions}

It has been reported that the application of mesoporous silica as a carrier for poorly watersoluble drugs enhanced their solubility and dissolution rate (Sanganwar, 2008). The data presented shows that combining mesoporous silica with fenofibrate increased the rate at which fenofibrate dissolves. The results were most pronounced in the fusion mixtures.

It is clear from my work that combining fenofibrate with mesoporous silica altered the solid-state properties of fenofibrate. The crystallinity (ordered structure) of fenofibrate was reduced significantly and in some completely reduced when combined with excess mesoporous silica; this was especially apparent in the fusion/melting samples.

I wish to acknowledge help and support of all the staff of the School of Pharmacy (SOP) and especially my supervisors, Dr. Abina Crean and Dr. Katie Ryan. I would like also to thank the Solid State Pharmaceutical Cluster (SSPC) and Science Foundation Ireland (SFI) for providing funding for my PhD. Robert J. Ahern, Pharmaceutics, School of Pharmacy, UCC; Abina M. Crean, Pharmaceutics, School of Pharmacy and Analytical and Biological Chemistry Research Facility UCC; and Katie Ryan, School of Pharmacy, UCC. 